a-Cyano-4-Hydroxycinnamic Acid and Tri-Potassium Citrate Salt Pre-Coated

Silicon Nanopost Array Provides Enhanced Lipid Detection for High Spatial

\title{
Resolution MALDI Imaging Mass Spectrometry
}

\section{Authors}

Martin Dufresne ${ }^{1,2}$, Jarod A. Fincher ${ }^{1,2}$, Nathan H. Patterson ${ }^{1,2}$, Kevin L. Schey ${ }^{1,2,3}$, Jeremy L. Norris ${ }^{1,2,3}$, Richard M. Caprioli ${ }^{1,2,3,4}$, and Jeffrey M. Spraggins ${ }^{1,2,3,5^{*}}$.

\section{Affiliations}

${ }^{1}$ Mass Spectrometry Research Center, Vanderbilt University, Nashville, TN 37235, USA

${ }^{2}$ Department of Biochemistry, Vanderbilt University, Nashville, TN 37205, USA

${ }^{3}$ Department of Chemistry, Vanderbilt University, Nashville, TN 37235, USA

${ }^{4}$ Department of Pharmacology, Vanderbilt University, Nashville, TN 37232, USA

${ }^{5}$ Department of Cell \& Developmental Biology, Vanderbilt University, Nashville, TN 37232, USA

\section{*Corresponding author:}

Jeffrey M. Spraggins

jeff.spraggins@vanderbilt.edu

Keywords: Imaging mass spectrometry, MALDI, NAPA, sample preparation, potassium, phospholipids, triglycerides, cholesteryl ester, cerebroside, lipids, neutral lipids, FT-ICR, timsTOF 


\section{Table of Contents}

MALDI MS ion optic instrumental parameters

Discussion on NAPA vs pre-coated NAPA

Supplemental Table 1: M5 Sprayer parameters

Supplemental Table 2: H\&E staining protocol

Supplemental Table 3: MALDI MS parameters

Supplemental Table 4: Intensity, signal to noise value, and associated fold changes of different lipids

Figure S1: Rabbit adrenal gland and a mouse brain homogenate spectral comparison

Figure S2: Mouse liver and a rat testis homogenate spectral comparison

Figure S3: Lithium, Sodium, and Potassium citrate spectral comparison on mouse brain homogenate

Figure S4: Fold changes on various tissue homogenate of NAPA vs pre-coated NAPA ( $=3$ )

Figure S5: Ion images from mouse brain on NAPA

Figure S6: Collection of MALDI IMS images from an albino rat eye at 10 um lateral resolution

Figure S7: H\&E stained albino rat eye 


\section{MALDI ion optic instrumental parameters}

The additional prototype MALDI timsTOF Pro values include: an ion transfer time of $70 \mu$ s, a pre-pulse storage time of $5 \mu \mathrm{s}$, a collision RF of $1000 \mathrm{Vpp}$, a funnel 1 RF of $350 \mathrm{Vpp}$, a funnel $2 \mathrm{RF}$ of $400 \mathrm{Vpp}$, and a multipole RF of $500 \mathrm{Vpp}$.

The additional 9.4T FT-ICR values include: an ion transfer time of $0.7 \mathrm{~ms}$, a transfer optic frequency of $4 \mathrm{MHz}$, a transfer optic RF amplitude of $380 \mathrm{Vpp}$, an octupole frequency of $5 \mathrm{MHz}$, an octupole RF amplitude of 400 Vpp, and a data size of $512 \mathrm{k}$ creating a $0.3146 \mathrm{~s}$ transient leading to a resolving power of $\sim 40000$ at $\mathrm{m} / \mathrm{z}$ 866.649 .

The additional 15T FT-ICR values include: an ion transfer time of $0.75 \mathrm{~ms}$, a transfer optic frequency of 4 $\mathrm{MHz}$, a transfer optic RF amplitude of $350 \mathrm{Vpp}$, an octupole frequency of $5 \mathrm{MHz}$, an octupole RF amplitude of $350 \mathrm{Vpp}$, a collision RF amplitude of $2000 \mathrm{Vpp}$, and a data size of $256 \mathrm{k}$ creating a $0.5767 \mathrm{~s}$ transient leading to a resolving power of $\sim 90000$ at $m / z 866.649$.

\section{NAPA vs pre-coated NAPA}

The reason for increases between phospholipids and neutral lipids using pre-coated NAPA compared to bare NAPA is two-fold. First, NAPA induced phospholipid fragmentation is reduced with the lower laser power required for proper desorption and ionization of the pre-coated chips along with CHCA buffering the heat exchange process between the nanopost and the tissue leading to lower in-source fragmentation. Second, in tissues with high neutral lipid content the phospholipids experience ion suppression effects due to their lower affinity for metal cations. This can be seen in the adrenal gland homogenate where, on NAPA, the average sum signal for PEs is roughly $1 / 12$ of the CEs average sum signal and with the pre-coated NAPA substrate the PEs are roughly at half the average sum signal of the CEs (Figure S3). This change is in part due to the lower fragmentation but also due to the addition of potassium salt which alleviates ion suppression by introducing more available potassium cations in the desorption plume. 
Supplemental Table 1: M5 Sprayer parameters

\begin{tabular}{|c|c|c|c|c|c|c|c|c|}
\hline Matrix & $\begin{array}{c}\text { Temperatures } \\
\left({ }^{\circ} \mathrm{C}\right)\end{array}$ & $\begin{array}{l}\text { Concentration } \\
(\mathrm{mg} / \mathrm{mL})\end{array}$ & $\begin{array}{c}\text { Spray } \\
\text { speed } \\
(\mathrm{mm} / \mathrm{min})\end{array}$ & $\begin{array}{l}\text { Flow rate } \\
(\mathrm{mL} / \mathrm{min})\end{array}$ & $\begin{array}{c}\mathrm{N}_{2} \\
\text { pressure } \\
(\mathrm{psi})\end{array}$ & $\begin{array}{c}\# \\
\text { passes }\end{array}$ & $\begin{array}{l}\text { Spacing } \\
\text { (mm) }\end{array}$ & $\begin{array}{l}\text { Spray } \\
\text { pattern }\end{array}$ \\
\hline \multirow[b]{2}{*}{$\mathrm{CHCA}$} & 70 nozzle & \multirow[b]{2}{*}{5.6} & \multirow[b]{2}{*}{1350} & \multirow[b]{2}{*}{0.05} & \multirow[b]{2}{*}{10} & \multirow[b]{2}{*}{4} & \multirow[b]{2}{*}{2} & \multirow[b]{2}{*}{$\mathrm{HH}$} \\
\hline & $\begin{array}{c}55 \text { heating } \\
\text { stage }\end{array}$ & & & & & & & \\
\hline \multirow[b]{2}{*}{ DAN } & 40 nozzle & \multirow[b]{2}{*}{10} & \multirow[b]{2}{*}{1200} & \multirow[b]{2}{*}{0.1} & \multirow[b]{2}{*}{10} & \multirow[b]{2}{*}{6} & \multirow[b]{2}{*}{3} & \multirow[b]{2}{*}{$\mathrm{HH}$} \\
\hline & $\begin{array}{l}55 \text { heating } \\
\text { stage }\end{array}$ & & & & & & & \\
\hline \multirow[b]{2}{*}{ DHB } & 70 nozzle & \multirow[b]{2}{*}{20} & \multirow[b]{2}{*}{1350} & \multirow[b]{2}{*}{0.05} & \multirow[b]{2}{*}{10} & \multirow[b]{2}{*}{4} & \multirow[b]{2}{*}{1.5} & \multirow[b]{2}{*}{$\mathrm{HH}$} \\
\hline & $\begin{array}{l}55 \text { heating } \\
\text { stage }\end{array}$ & & & & & & & \\
\hline \multirow[b]{2}{*}{ DHA } & 70 nozzle & \multirow[b]{2}{*}{15} & \multirow[b]{2}{*}{1300} & \multirow[b]{2}{*}{0.1} & \multirow[b]{2}{*}{10} & \multirow[b]{2}{*}{4} & \multirow[b]{2}{*}{2} & \multirow[b]{2}{*}{$\mathrm{HH}$} \\
\hline & $\begin{array}{l}55 \text { heating } \\
\text { stage }\end{array}$ & & & & & & & \\
\hline
\end{tabular}

Supplemental Table 2: H\&E staining protocol

\begin{tabular}{|c|c|}
\hline \multicolumn{2}{|c|}{ H\&E staining protocol } \\
\hline Steps (immersion) & Time \\
\hline $95 \%$ EtOH & $30 \mathrm{~s}$ \\
\hline $70 \%$ EtOH & $30 \mathrm{~s}$ \\
\hline Water & $30 \mathrm{~s}$ \\
\hline Hematoxylin & $2.5 \mathrm{~min}$ \\
\hline Water & $20 \mathrm{~s}$ \\
\hline $70 \%$ EtOH & $30 \mathrm{~s}$ \\
\hline $95 \%$ EtOH & $30 \mathrm{~s}$ \\
\hline Eosin & $1 \mathrm{~min}$ \\
\hline $95 \%$ EtOH & $30 \mathrm{~s}$ \\
\hline $100 \%$ EtOH & $30 \mathrm{~s}$ \\
\hline Xylene & $5 \mathrm{~min}$ \\
\hline
\end{tabular}

Hematoxylin stock solution was prepared following the Carazzi method. In short, $1 \mathrm{~g}$ of hematoxylin along with $45.93 \mathrm{~g}$ of potassium alum and $0.1 \mathrm{~g}$ of sodium iodate were dissolved in $400 \mathrm{~mL}$ of water and $100 \mathrm{~mL}$ of glycerol. The final solution was filtered through a Whatman paper \#1. Eosin stock solution was purchased from (RichardAllan Sci \#7111). $50 \mathrm{~mL}$ of stock eosin was mixed with $0.57 \mathrm{~mL}$ of Pholoxine B at $1 \%$ in water. 
Supplemental Table 3: MALDI MS instrumental parameters

\begin{tabular}{|c|c|c|c|c|c|c|}
\hline Instruments & Laser type & $\begin{array}{c}\text { Laser } \\
\text { frequency }\end{array}$ & Laser focus & $\begin{array}{c}\text { Absolute } \\
\text { laser } \\
\text { power }(\%)\end{array}$ & $\begin{array}{c}\text { Spot } \\
\text { size } \\
(\mu \mathrm{m})\end{array}$ & Comments \\
\hline timsTOF & $\begin{array}{c}\text { Smartbeam } \\
3 \mathrm{D} \\
\text { Gaussian }\end{array}$ & 1000 & $\begin{array}{c}\text { Custom no } \\
\text { beamscan }\end{array}$ & 23 & $\sim 8 \pm 1$ & $\begin{array}{c}\mathrm{m} / \mathrm{z} 500-2000 \\
25 \text { shots/pixel }\end{array}$ \\
\hline $\begin{array}{c}\text { SolariX } \\
15 \mathrm{~T}\end{array}$ & Smartbeam II & 500 & minimum & 35 & $\sim 24 \pm 2$ & $\begin{array}{c}\mathrm{m} / \mathrm{z} 500-1500 \\
50 \text { shots } / \text { pixel }\end{array}$ \\
\hline $\begin{array}{c}\text { SolariX } \\
9.4 \mathrm{~T}\end{array}$ & $\begin{array}{c}\text { Modified } \\
\text { Smartbeam II } \\
\text { Gaussian }\end{array}$ & 500 & minimum & 16 & $\sim 18 \pm 2$ & $\begin{array}{c}\mathrm{m} / \mathrm{z} 350-1500 \\
50 \text { shots } / \text { pixel }\end{array}$ \\
\hline
\end{tabular}

Supplemental Table 4: Intensity, signal to noise value, and associated fold changes as provided by mMass between NAPA and pre-coated NAPA for the highest intensity signal found in pre-coated NAPA of each detected lipid classes from the fold change experiment of a mouse brain tissue homogenate using a Solarix 9.4T MALDI FT-ICR instrument ( $\mathrm{n}=3)$.

\begin{tabular}{|c|c|c|c|c|}
\hline & \multirow{3}{*}{$\frac{\text { NAPA }}{7.3 \mathrm{e} 6 \pm 1 \mathrm{e} 5}$} & \multirow{3}{*}{$\begin{array}{c}\text { Pre-coated NAPA } \\
3.8 \mathrm{e} 7 \pm 1 \mathrm{e} 6\end{array}$} & \multirow{3}{*}{$\begin{array}{c}\text { Fold Changes } \\
5.2 \pm 0.2\end{array}$} \\
\hline & & & & \\
\hline \multirow{2}{*}{$\begin{array}{c}\operatorname{PA}(36: 2) \\
m / z, 739.468\end{array}$} & Intensity (a.u.) & & & \\
\hline & $\mathbf{S} / \mathbf{N}$ & $7090 \pm 160$ & $44200 \pm 3400$ & $5.8 \pm 0.6$ \\
\hline \multirow{2}{*}{$\begin{array}{c}\text { PE(40:6) } \\
\mathrm{m} / \mathrm{z} 868.465\end{array}$} & Intensity (a.u.) & $4,0190 \mathrm{e} 6 \pm 6 \mathrm{e} 3$ & $2.640 \mathrm{e} 7 \pm 6 \mathrm{e} 5$ & $6.6 \pm 0.1$ \\
\hline & $\mathbf{S} / \mathbf{N}$ & $4010 \pm 40$ & $29100 \pm 1700$ & $7.3 \pm 0.5$ \\
\hline \multirow{2}{*}{$\begin{array}{c}\text { PE(p40:6) } \\
\text { m/z 814.515 }\end{array}$} & Intensity (a.u.) & $1.44 \mathrm{e} 6 \pm 2 \mathrm{e} 4$ & $1.14 \mathrm{e} 7 \pm 5 \mathrm{e} 5$ & $7.9 \pm 0.2$ \\
\hline & $\mathbf{S} / \mathbf{N}$ & $1460 \pm 30$ & $13000 \pm 900$ & $8.9 \pm 0.7$ \\
\hline \multirow{2}{*}{$\begin{array}{c}\mathrm{PC}(34: 2) \\
\mathrm{m} / \mathrm{z} 798.541\end{array}$} & Intensity (a.u.) & $1.96 \mathrm{e} 6 \pm 2 \mathrm{e} 4$ & $1.03 \mathrm{e} 7 \pm 2 \mathrm{e} 5$ & $5.3 \pm 0.1$ \\
\hline & $\mathbf{S} / \mathbf{N}$ & $2300 \pm 500$ & $11900 \pm 900$ & $5 \pm 1$ \\
\hline \multirow{2}{*}{$\begin{array}{c}\text { HexCer(42:1;O3) } \\
\text { m/z 866.648 }\end{array}$} & Intensity (a.u.) & $6,361 \mathrm{e} 6 \pm 8 \mathrm{e} 4$ & $1,864 \mathrm{e} 7 \pm 5 \mathrm{e} 5$ & $2.9 \pm 0.1$ \\
\hline & $\mathbf{S} / \mathbf{N}$ & $6360 \pm 80$ & $20500 \pm 1400$ & $3.2 \pm 0.3$ \\
\hline \multirow{2}{*}{$\begin{array}{c}\text { PI(38:4) } \\
\mathrm{m} / \mathrm{z} \text { 963.475 }\end{array}$} & Intensity (a.u.) & $2.7 \mathrm{e} 5 \pm 2 \mathrm{e} 4$ & $4.76 \mathrm{e} 6 \pm 5 \mathrm{e} 4$ & $18 \pm 1$ \\
\hline & $\mathbf{S} / \mathbf{N}$ & $250 \pm 20$ & $5100 \pm 300$ & $20 \pm 2$ \\
\hline \multirow{2}{*}{$\begin{array}{c}\text { PI-Cer(40:0) } \\
\text { m/z 904.604 }\end{array}$} & Intensity (a.u.) & $\mathrm{N} / \mathrm{D}$ & $7.5 \mathrm{e} 5 \pm 2 \mathrm{e} 4$ & $\varnothing$ \\
\hline & $\mathbf{S} / \mathbf{N}$ & N/D & $830 \pm 60$ & $\varnothing$ \\
\hline \multirow{2}{*}{$\begin{array}{c}\operatorname{PS}(44: 3) \\
\mathrm{m} / \mathrm{z} 974.564\end{array}$} & Intensity (a.u.) & $6.3 \mathrm{e} 4 \pm 8 \mathrm{e} 3$ & $3.92 \mathrm{e} 6 \pm 6 \mathrm{e} 4$ & $62 \pm 7$ \\
\hline & $\mathbf{S} / \mathbf{N}$ & $250 \pm 20$ & $1940 \pm 140$ & $8 \pm 1$ \\
\hline \multirow{2}{*}{$\begin{array}{c}\text { SulfoHexCer(42:2;O2) } \\
\mathrm{m} / \mathrm{z} 966.551\end{array}$} & Intensity (a.u.) & $2.6 \mathrm{e} 5 \pm 2 \mathrm{e} 4$ & $1.80 \mathrm{e} 6 \pm 4 \mathrm{e} 4$ & $6.9 \pm 0.6$ \\
\hline & $\mathbf{S} / \mathbf{N}$ & $250 \pm 20$ & $1940 \pm 140$ & $8 \pm 1$ \\
\hline
\end{tabular}




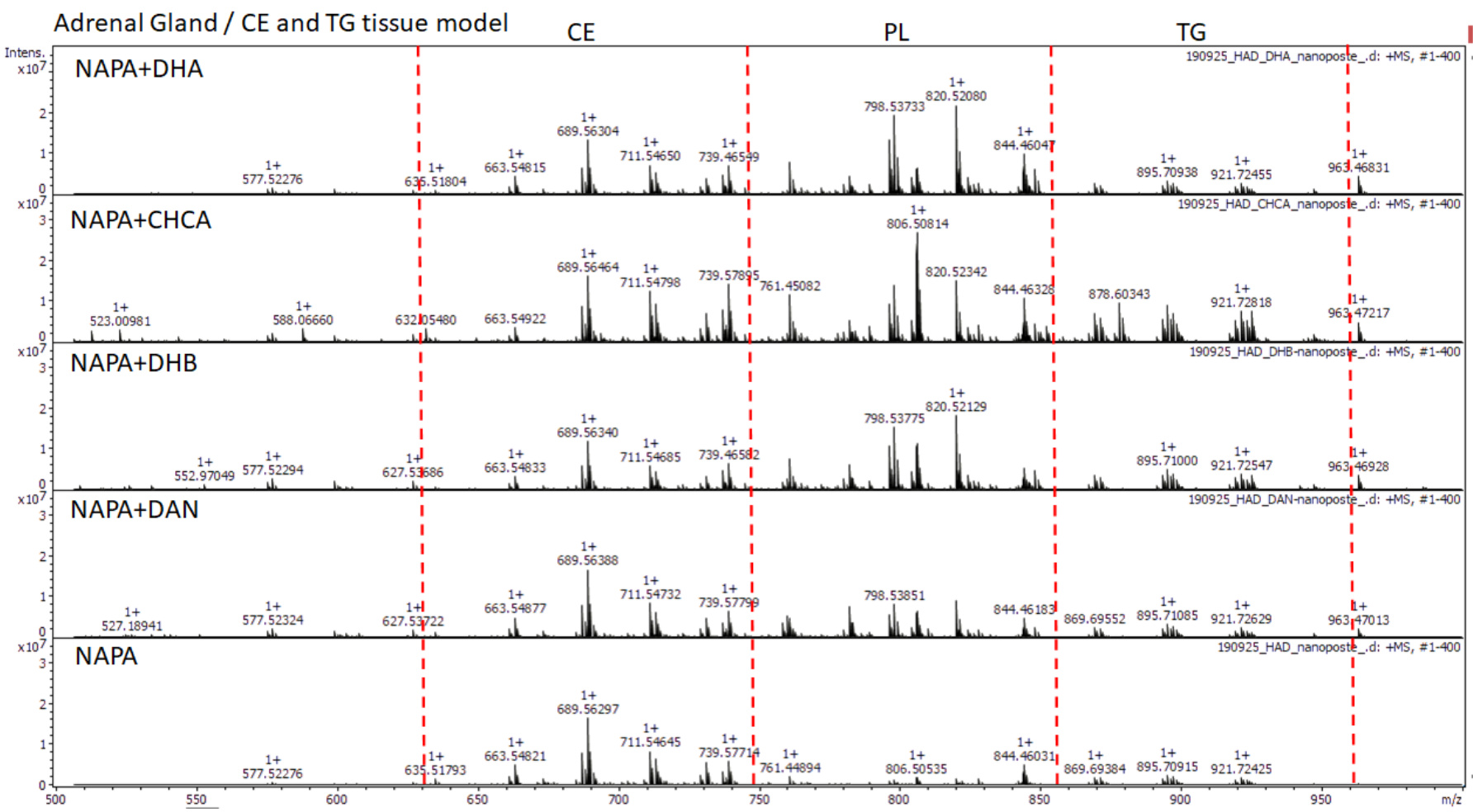

Mouse Brain / HexCer tissue model

PL and HexCer

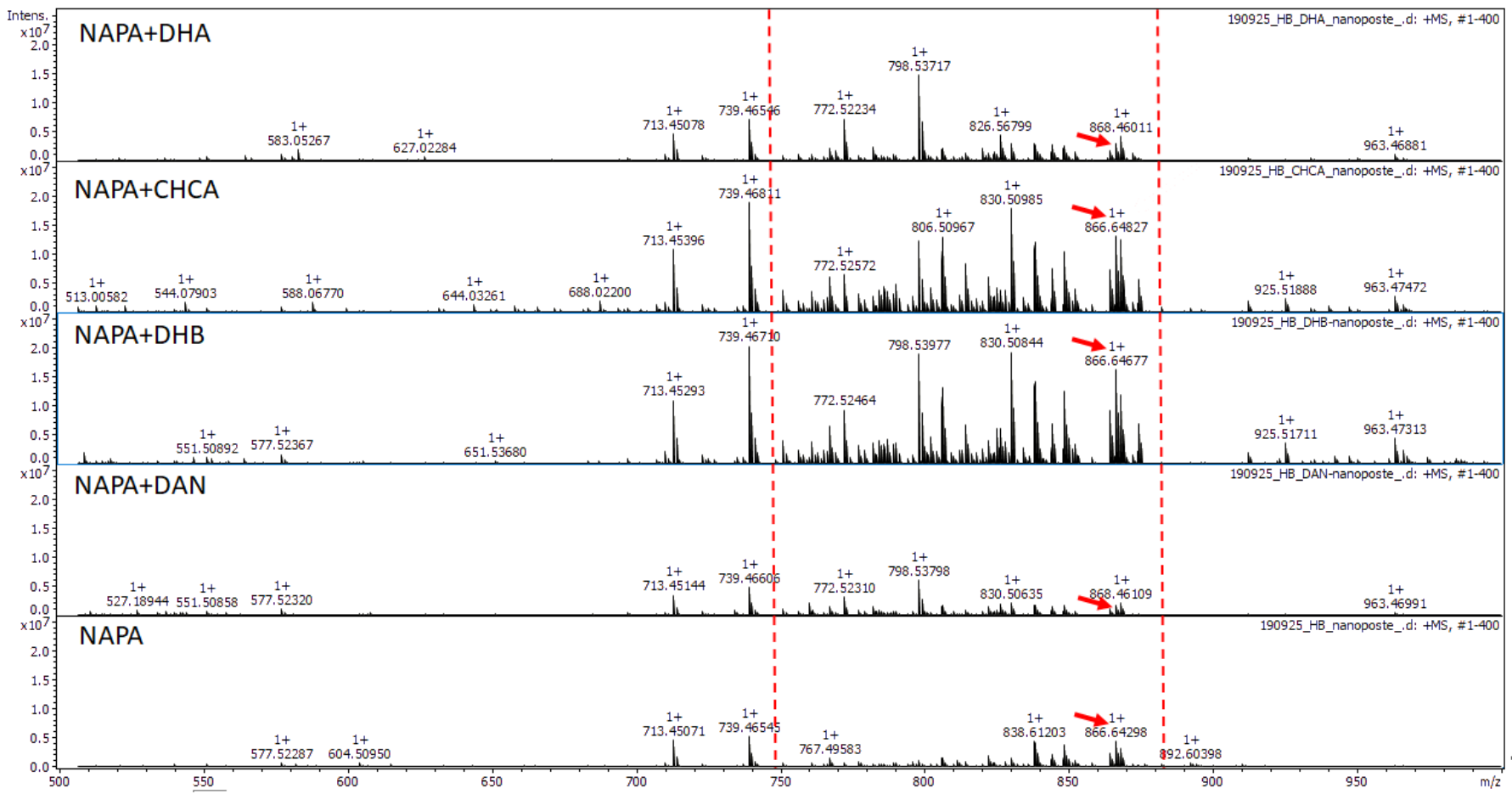

Figure S1: Average spectra of 400 pixels from a rabbit adrenal gland and a mouse brain homogenate on NAPA or pre-coated NAPA with various matrices acquired with a 15T FT-ICR in positive mode. DHA showed poor HexCer ionization in the brain homogenate as highlighted by the red arrow pointing to $\operatorname{Hex} \operatorname{Cer}(42: 1 ; \mathrm{O} 3)$ at $\mathrm{m} / \mathrm{z}$ 688.6468. In both homogenate both CHCA and DHB show the best signal intensities and number of detected signals for phospholipids (PL) and neutral lipids. The red dotted lines are to emphasis the typical mass region where certain classes of lipids can be found. 

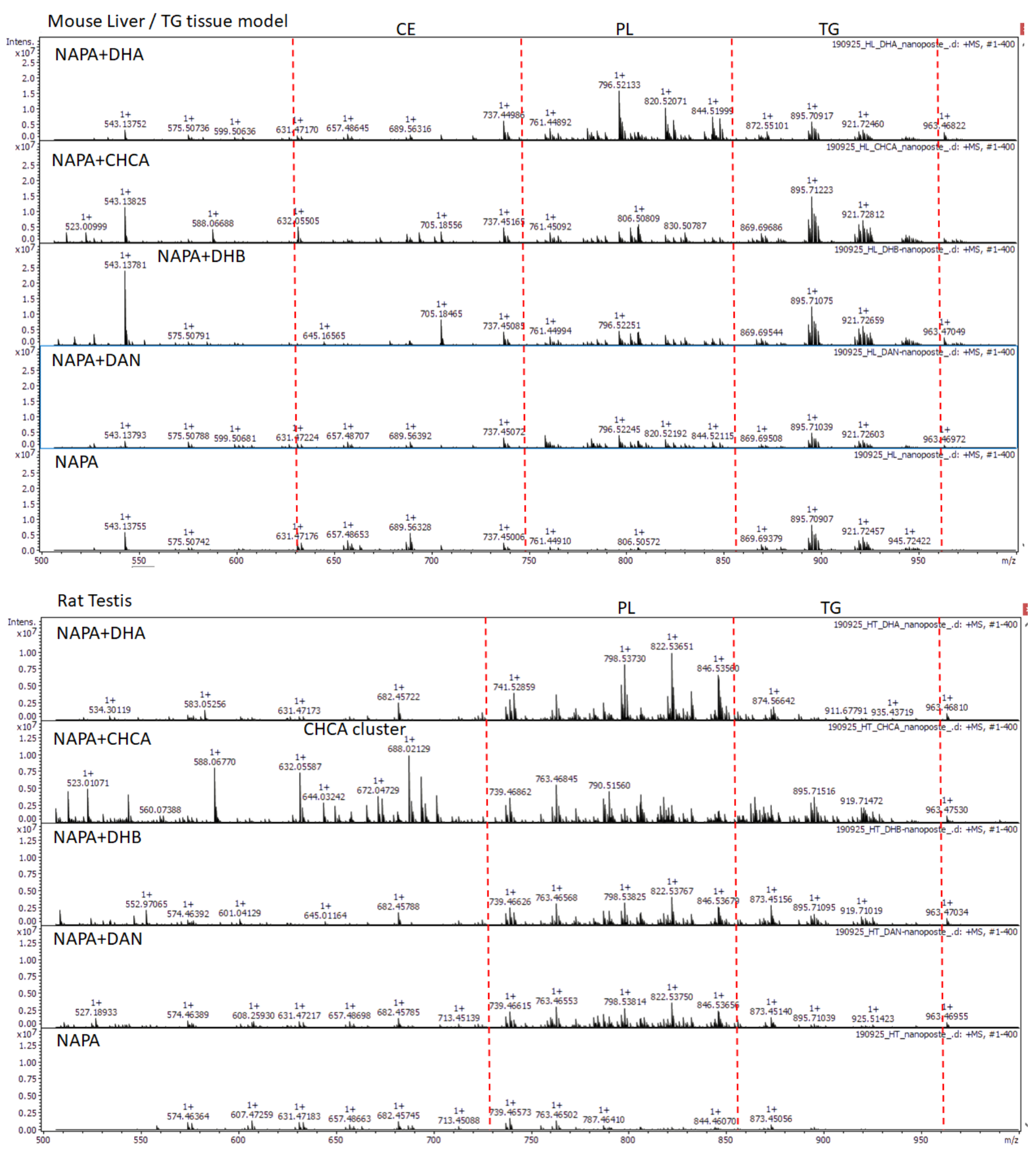

Figure S2: Average spectra of 400 pixels from a mouse liver and a rat testis homogenate on NAPA or precoated NAPA with various matrices acquired with a $15 \mathrm{~T} \mathrm{FT-ICR} \mathrm{in} \mathrm{positive} \mathrm{mode.} \mathrm{In} \mathrm{both} \mathrm{homogenate} \mathrm{both}$ CHCA and DHB show the best number of detected signals for phospholipids (PL) and neutral lipids. Here DHA surpasses both matrices when it comes to intensities but fails to properly ionize neutral lipids. The red dotted lines are to emphasis the typical mass region where certain classes of lipids can be found. 


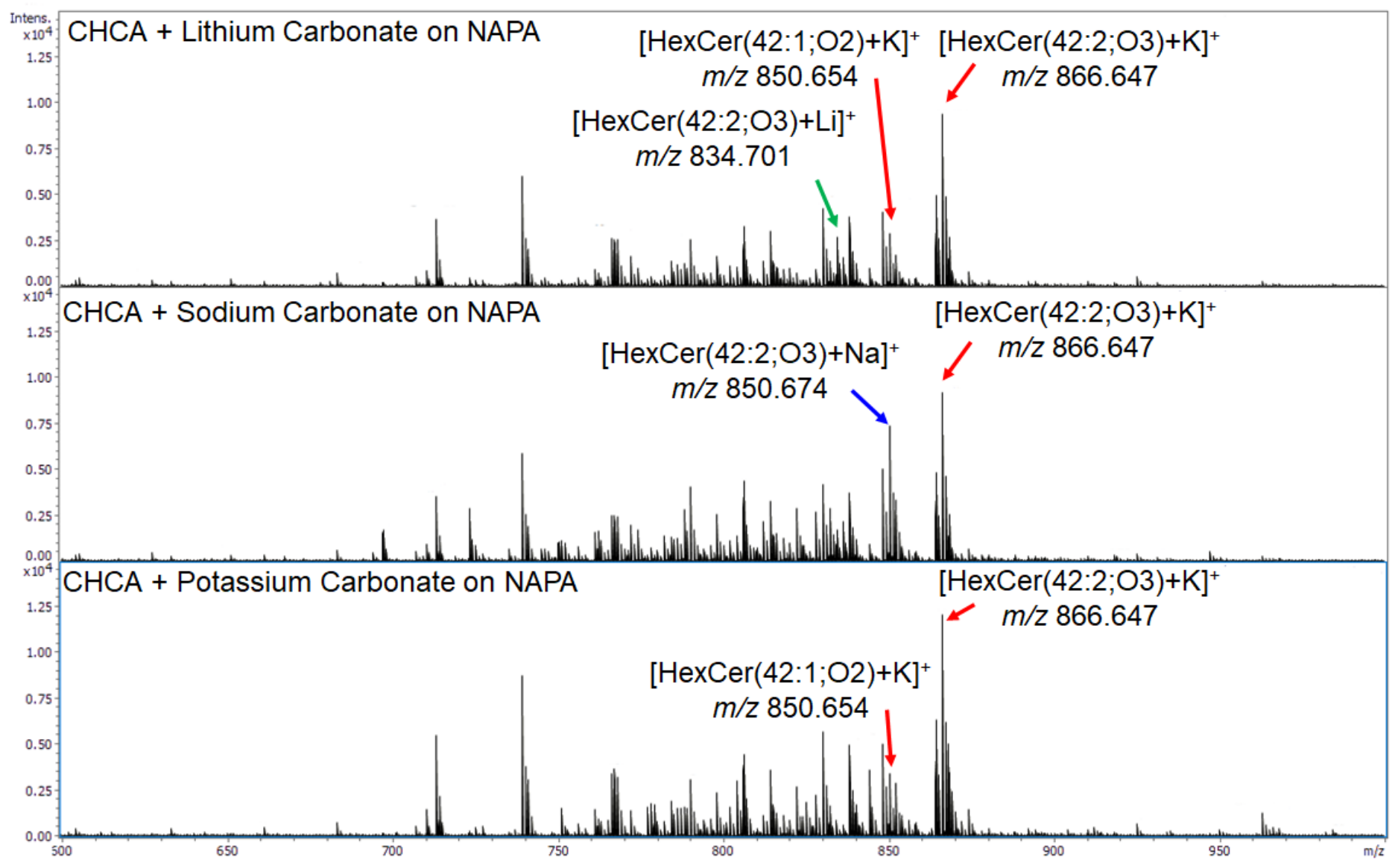

Figure S3: Average spectra of 400 pixels from a mouse brain homogenate on pre-coated NAPA with different alkali metal salts acquire on a prototype MALDI timsTOF PRO. In both the lithium and sodium pre-coating experiments, the base peak remains the potassiated HexCer(42:2;O3) and most of the detected PL remain as potassium adduct over lithium or sodium adducts. In the potassium doping experiment, neither the $[\mathrm{M}+\mathrm{Li}]^{+}$or $[\mathrm{M}+\mathrm{Na}]^{+}$adduct were detected for $\operatorname{HexCer}(42: 2 ; \mathrm{O} 3)$ or for any other detected lipids. 


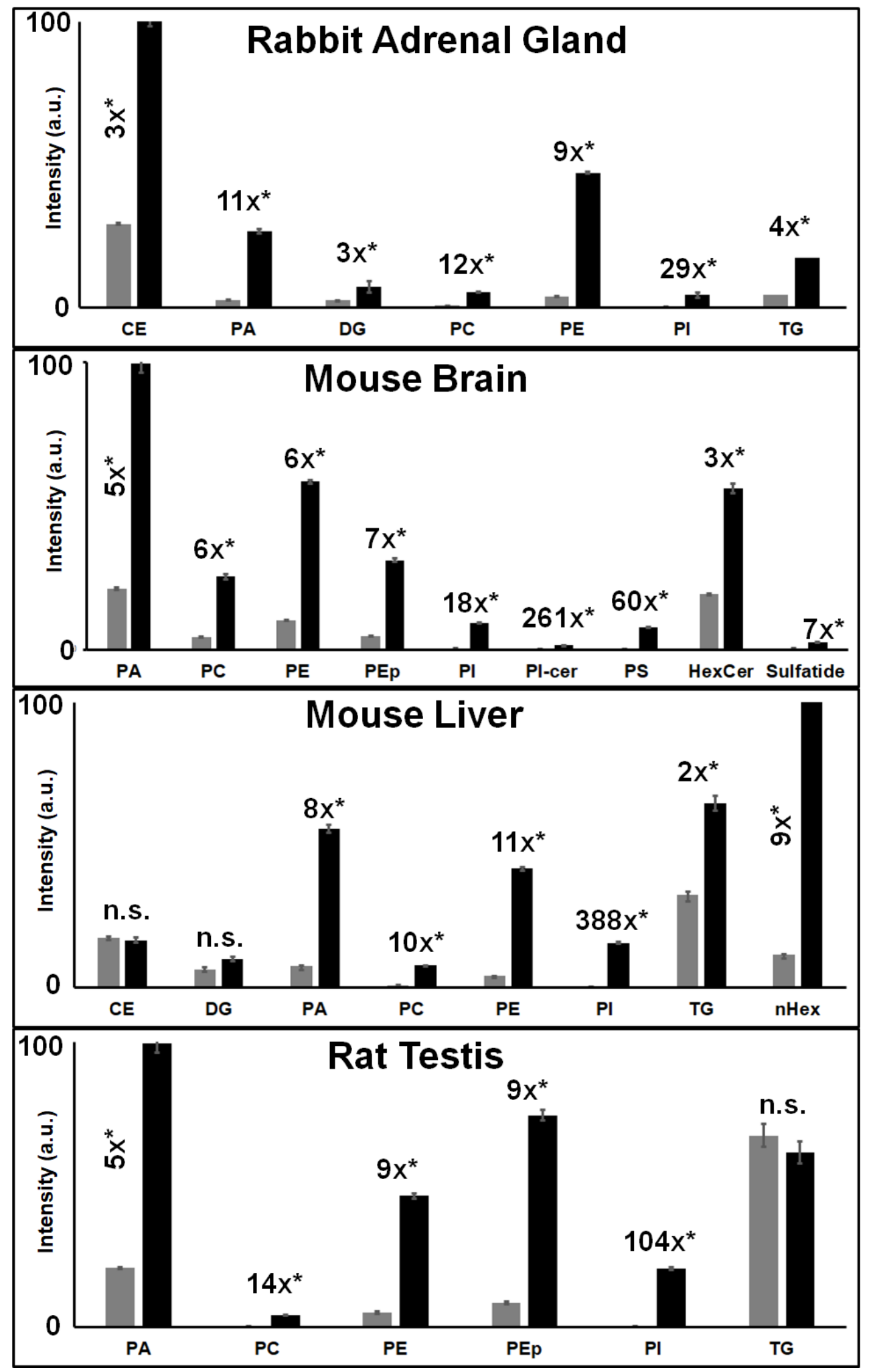

Figure S4: Bar graph representation of lipid signal fold increase on various tissue homogenate from the summation of the three most abundant signals for each lipid classes $(n=3)$. Fold increases for phospholipids that are not in-source fragments range from 6 up to 591. Fold change for neutral lipids is lower with some being not significant (n.s.) or with significance being reached from 2 to 3 fold increases.

$*$ p-value $\leq 0.05$ 


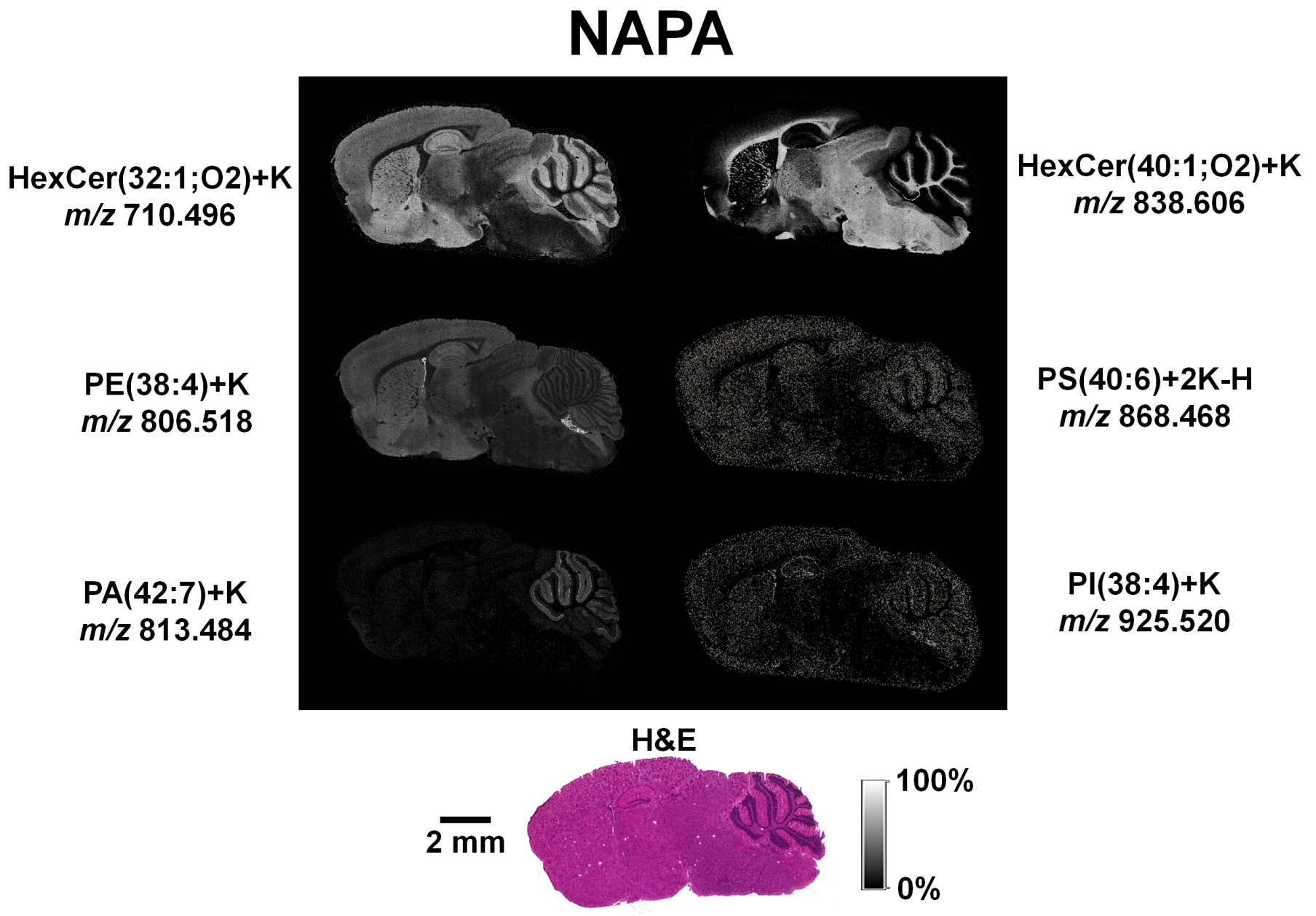

Figure S5: Ion images from mouse brain on NAPA at a spatial resolution of $25 \mu \mathrm{m}$ with their intensities not normalized to pre-coated NAPA. Pre-coated NAPA does produce higher signal intensity and allows detection of more phospholipids but NAPA without pre-coating is still capable of visualizing most of these lipids at a low laser power. 


\section{[Cholesterol+K] ${ }^{\mathbf{}}$}

$\mathrm{m} / \mathrm{z} 425.3163 \pm 6.5 \mathrm{mDa}$ 4 ppm
Intensity (arb. unit) $\widehat{0} \%$

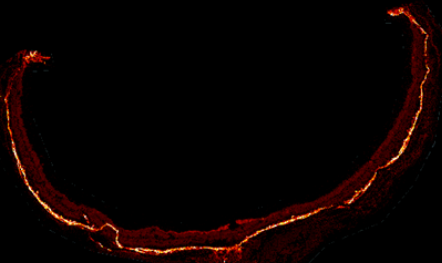

[HexCer(34:1;02)+K] ${ }^{+}$Intensity (arb. unit) $\mathrm{m} / \mathrm{z} 740$
$1 \mathrm{ppm}$ 0\% $208 \%$
[PE(p-34:1)+K]+ $\mathrm{m} / \mathrm{z} 740.4995 \pm 6.5 \mathrm{mDa}$ $1 \mathrm{ppm}$

Intensity (arb. unit) $0 \%$

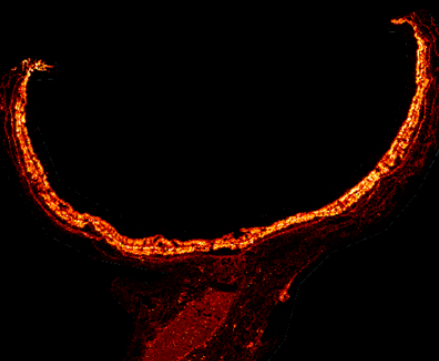

[PC(32:0)+K]+

Intensity (arb. unit)

[PE(p-36:1)+K] ${ }^{+} \quad$ Intensity (arb. unit) $10 \%$ $186 \% \quad \mathrm{~m} / \mathrm{z} 772.5233 \pm 6.5 \mathrm{mDa}$ . 2 ppm

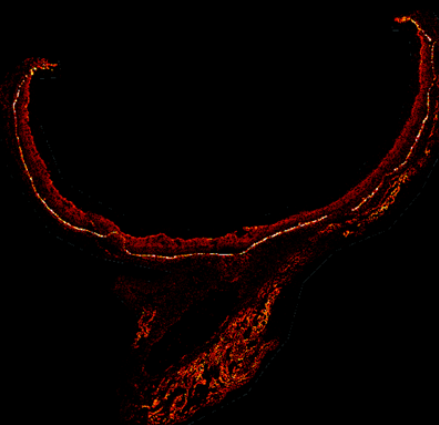

Intensity (arb. unit) $\widehat{0} \%$

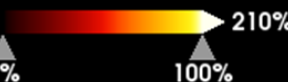

\section{[PE(36:4)+K] +}

$\mathrm{m} / \mathrm{z} 778.4815 \pm 6.5 \mathrm{mDa}$ 4 ppm

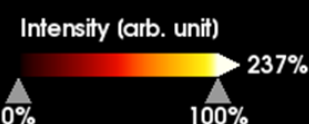

$0 \%$

$100 \%$
$0 \%$

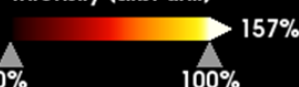
$100 \%$ $241 \%$

[PA(36:2)-H+2K] ${ }^{+}$ $\mathrm{m} / \mathrm{z} 777.4248 \pm 6.5 \mathrm{mDa}$ $2 \mathrm{ppm}$

$[P E(p-36: 4)+K]^{+}$
$m / z 762.4815 \pm 6.5 \mathrm{mDa}$

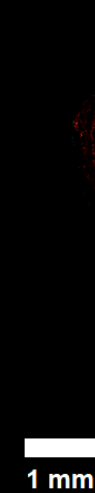

[PE(36:2)+K] +

$\mathrm{m} / \mathrm{z} 782.5079 \pm 6.5 \mathrm{mDa}$

$2 \mathrm{ppm}$

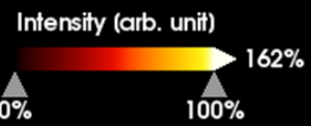

\begin{tabular}{|c|c|}
\hline$[\mathrm{PE}(36: 1)+\mathrm{K}]^{+}$ & Intensity (arb. unit) \\
\hline $\mathrm{m} / \mathrm{z} 784.5234 \pm 6.5 \mathrm{mDa}$ & \\
\hline
\end{tabular}

[PE(36:3)+K] +

$\mathrm{m} / \mathrm{z} 780.4952 \pm 6.5 \mathrm{mDa}$ $2 \mathrm{ppm}$

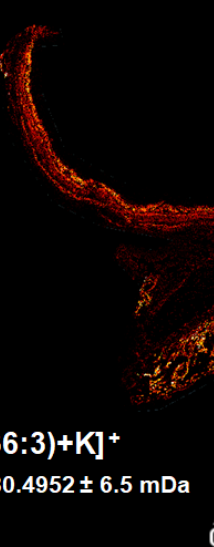

Intensity (arb. unit) $0 \%$

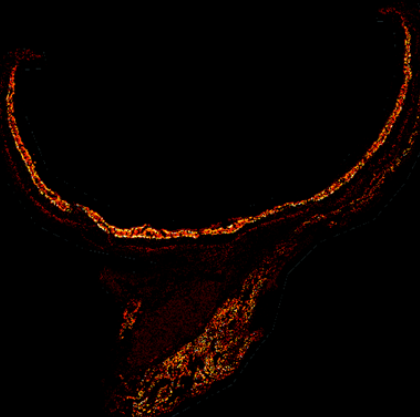

[PE(p-38:6)+K] ${ }^{+}$

Intensity (arb. unit) $\mathrm{m} / \mathrm{z} 786.4831 \pm 6.5 \mathrm{mDa}$ $0.4 \mathrm{ppm}$ A\% 


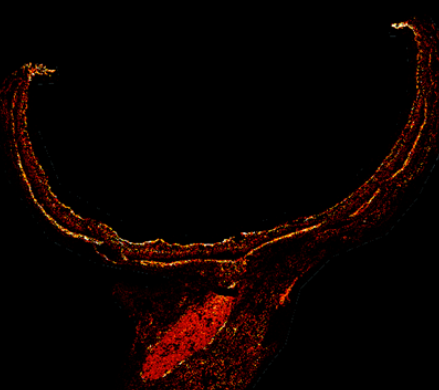

[PE(p-38:5)+K] +

$\mathrm{m} / \mathrm{z} 788.4962 \pm 6.5 \mathrm{mDa}$

$2 \mathrm{ppm}$
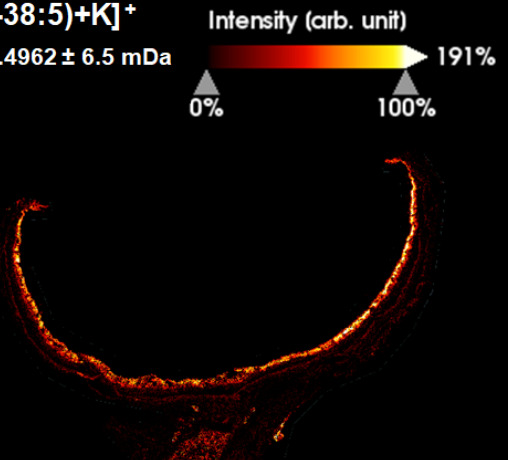

[PC(34:1)+K] +

$\mathrm{m} / \mathrm{z} 798.5414 \pm 6.5 \mathrm{mDa}$

$1 \mathrm{ppm}$

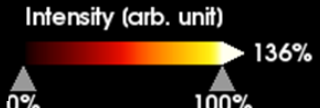

$0 \%$

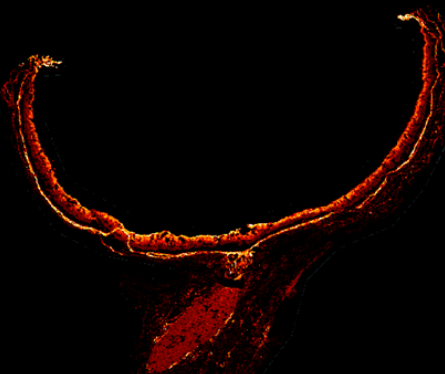

[PE(p-38:4)+K] ${ }^{+}$ $\mathrm{m} / \mathrm{z} 790.5167 \pm 6.5 \mathrm{mDa}$ $2 \mathrm{ppm}$
Intensity (arb. unit) $\hat{0} \%$

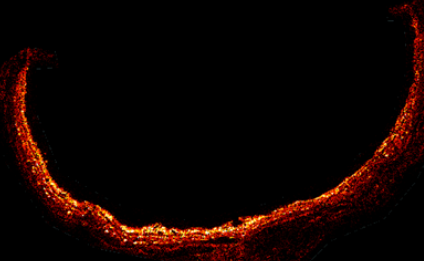

[PE(34:0)-H+2K] ${ }^{+}$

$195 \% \mathrm{~m} / \mathrm{z} 796.4679 \pm 6.5 \mathrm{mDa}$ $3 \mathrm{ppm}$

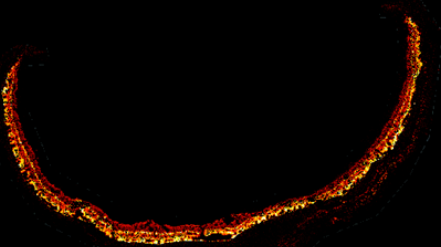

Intensity (arb. unit)

0\%

$168 \%$
[PE(p36:4)-H+2K] ${ }^{+}$Intensity (arb. unit) $\mathrm{m} / \mathrm{z} 800.4401 \pm 6.5 \mathrm{mDa}$ $1 \mathrm{ppm}$
$0 \%$

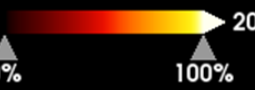

\section{[PE(38:6)+K] ${ }^{+}$}

$\mathrm{m} / \mathrm{z} 802.4762 \pm 6.5 \mathrm{mDa}$ 3 ppm
Intensity (arb. unit)

A\%

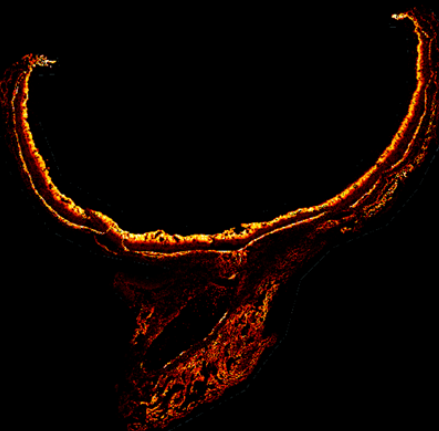

[PE(38:4)+K]+ Intensity (arb. unit) $\mathrm{m} / \mathrm{z} 806.5130 \pm 6.5 \mathrm{mDa}$ $4 \mathrm{ppm}$

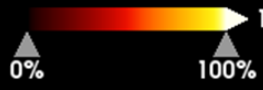
$100 \%$
[HexCer(38:1;03)+K] ${ }^{+}$Intensity (arb. unit) $\mathrm{m} / \mathrm{z} 810.5895 \pm 6.5 \mathrm{mDa}$ $5 \mathrm{ppm}$

0\%

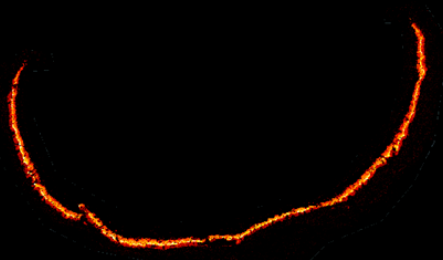

\section{$\mathrm{m} / \mathrm{z} 804.4725 \pm 6.5 \mathrm{mDa}$}

$2 \mathrm{ppm}$

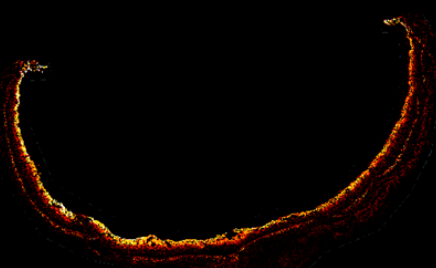

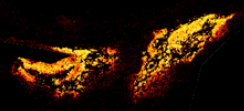

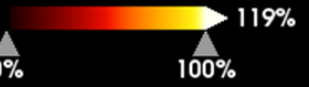

\section{$1 \mathrm{~mm}$}

$[\mathrm{PE}(\mathrm{p}-40: 7)+\mathrm{K}]^{+} \quad$ Intensity (arb. unit) $\mathrm{m} / \mathrm{z} 812.4955 \pm 6.5 \mathrm{mDa}$ $4 \mathrm{ppm}$

10\% $172 \%$

$[\mathrm{TG}(46: 2)+\mathrm{K}]^{+}$
$\mathrm{m} / \mathrm{z} 813.6402 \pm 6.5 \mathrm{mDa}$
$4 \mathrm{ppm}$
Intensity (arb. unit)

$\quad 100 \%$
[PE(p-40:6)+K] + $\mathrm{m} / \mathrm{z} 814.5159 \pm 6.5 \mathrm{mDa}$ $1 \mathrm{ppm}$
Intensity (arb. unit)

A\%
$121 \%$ 
$[\mathrm{PE}(\mathrm{p}-40: 5)+\mathrm{K}]^{+}$

$\mathrm{m} / \mathrm{z} 816.5268 \pm 6.5 \mathrm{mDa}$

4 ppm
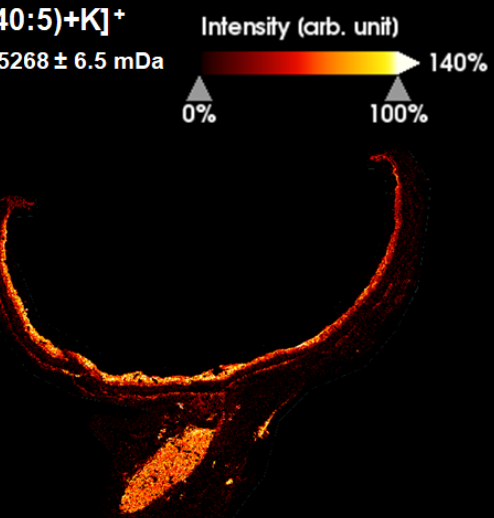

[PE(36:1)-H+2K] ${ }^{+}$

$\mathrm{m} / \mathrm{z} 822.4834 \pm 6.5 \mathrm{mDa}$

3 ppm

Intensity (arb. unit)

A

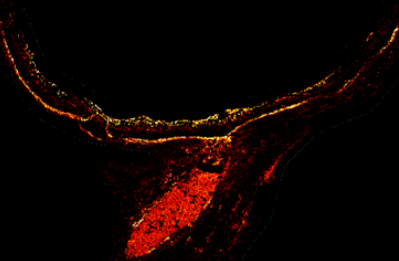

[PE(p-38:4)-H+2K] + Intensity (arb. unit)

$m / 2828.4750 \pm 6.5 \mathrm{mDa}$

$5 \mathrm{ppm}$
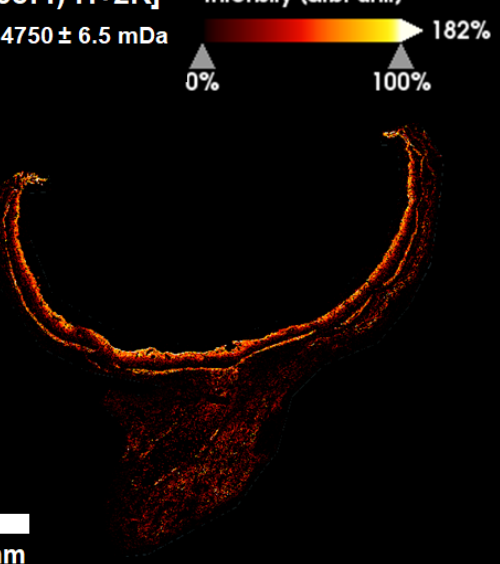

$$
1 \mathrm{~mm}
$$

[PC(34:2)-H+2K] ${ }^{+}$

$m / z 834.4840 \pm 6.5 \mathrm{mDa}$

3 ppm

\section{[PE(p-40:4)+K] +}

$\mathrm{m} / \mathrm{z} 818.5450 \pm 6.5 \mathrm{mDa}$

$1 \mathrm{ppm}$

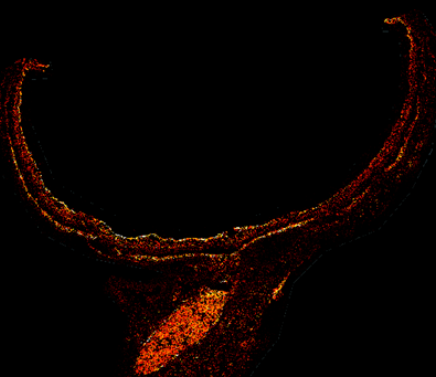

[PE(p-38:5)-H+2K] ${ }^{+}$Intensity (arb. unit) $\mathrm{m} / \mathrm{z} 826.4571 \pm 6.5 \mathrm{mDa}$ 3 ppm

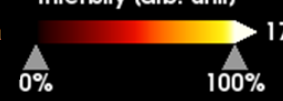
173

\section{[PE(36:2)-H+2K] + Intensity (arb. unit)} $\mathrm{m} / \mathrm{z} 820.4642 \pm 6.5 \mathrm{mDa}$ । 2 ppm $206 \%$ 今\% $100 \%$

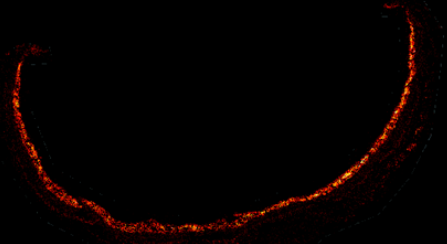

[PC(36:1)+K]+

$\mathrm{m} / \mathrm{z} 826.5717 \pm 6.5 \mathrm{mDa}$ $1 \mathrm{ppm} \quad 0 \%$

Intensity (arb. unit) A $180 \%$

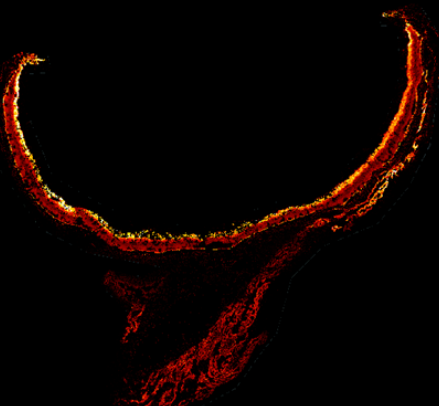

[PE(40:7)+K] + Intensity (arb. unit) $m / 2828.4908 \pm 6.5 \mathrm{mDa}$ $4 \mathrm{ppm}$
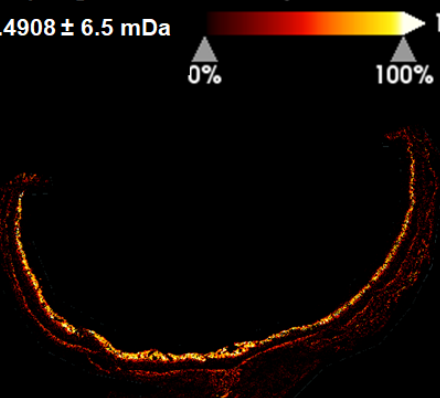

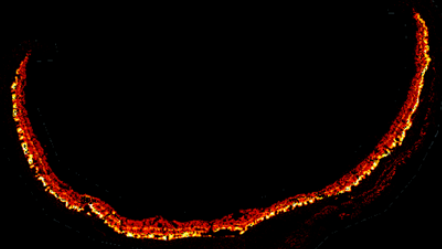




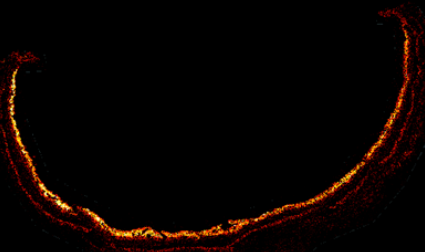

[PC(36:0)-H+2K1+ $\mathrm{m} / \mathrm{z} 838.5115 \pm 6.5 \mathrm{mDa}$ $1 \mathrm{ppm}$
Intensity (arb. unit) $0 \%$
[HexCer(40:1;03)+K] ${ }^{+}$Intensity (arb. unit) $159 \% \mathrm{~m} / \mathrm{z} 838.6233 \pm 6.5 \mathrm{mDa}$ $2 \mathrm{ppm}$

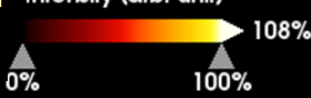

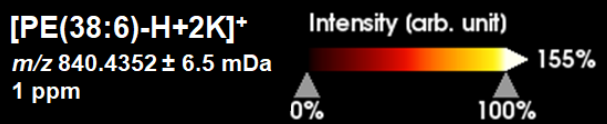

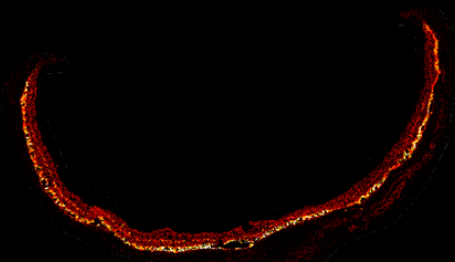

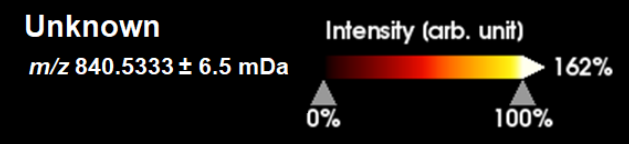

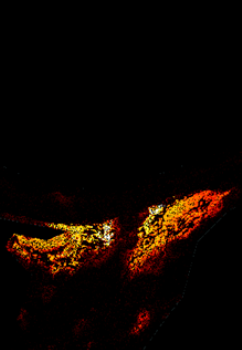

[TG(48:1)+K]+

$\mathrm{m} / \mathrm{z} 843.6842 \pm 6.5 \mathrm{mDa}$ $0.5 \mathrm{ppm}$

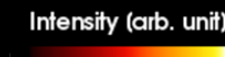

$0 \% \quad 100 \%$
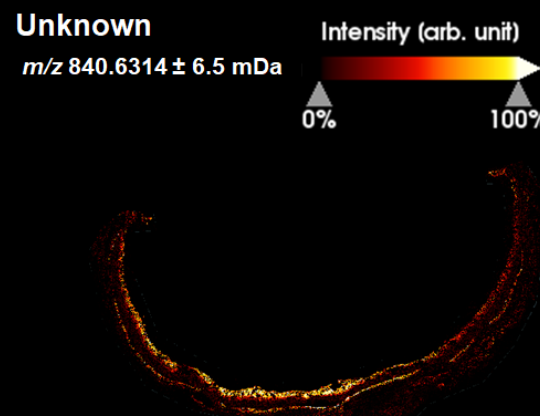

Intensity (arb. unit)

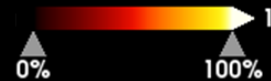

$0 \%$

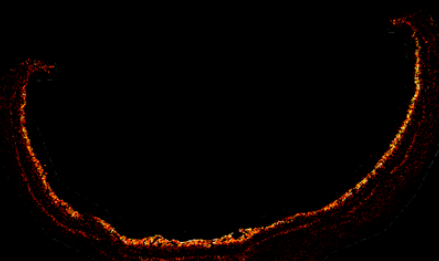

\section{Unknown}

$m / z 842.4968 \pm 6.5 \mathrm{mDa}$
Intensity (arb. unit)

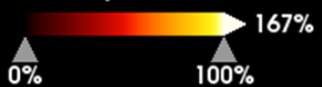

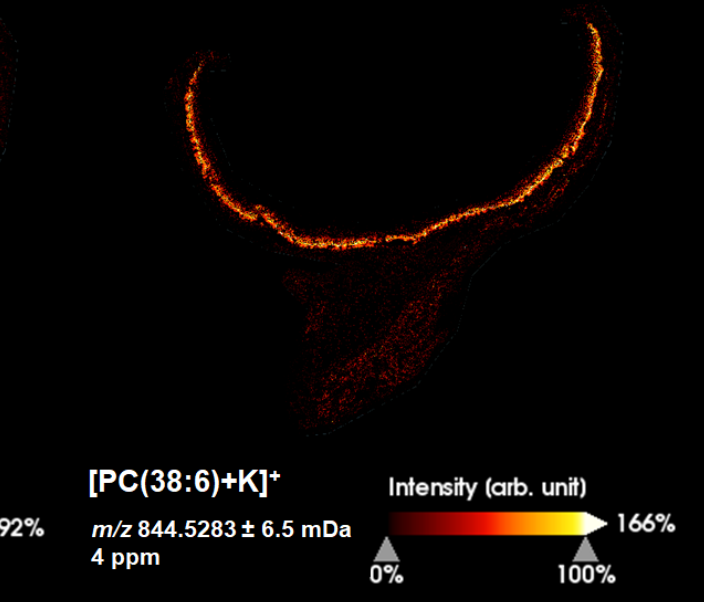

[PE(38:4)-H+2K] ${ }^{+}$

$3 \mathrm{ppm}$
Intensity (arb. unit)

$0 \%$

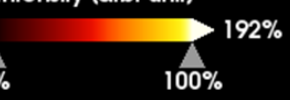

$[\mathrm{PC}(38: 6)+\mathrm{K}]^{+}$

4 ppm
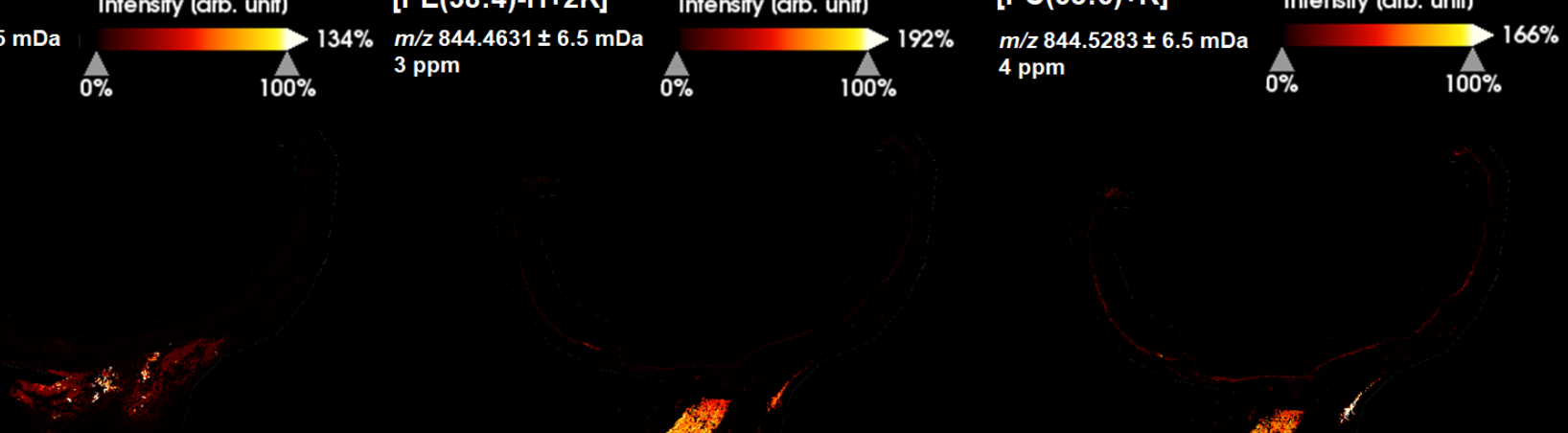

\section{$1 \mathrm{~mm}$}

\section{[TG(48:0)+K] $]^{+}$}

$\mathrm{m} / \mathrm{z} 845.6974 \pm 6.5 \mathrm{mDa}$

$2 \mathrm{ppm}$
Intensity (arb. unit) $288 \% \quad \mathrm{~m} / \mathrm{z} 848.6371 \pm 6.5 \mathrm{mDa}$ $0 \%$
$100 \%$
[HexCer(42:2;02)+K] ${ }^{+}$Intensity (arb. unit) $1 \mathrm{ppm}$
$0 \%$
[HexCer(42:1;02)+K]+ Intensity (arb. unit) $132 \% \mathrm{~m} / \mathrm{z} 850.6511 \pm 6.5 \mathrm{mDa}$

$3 \mathrm{ppm}$

$100 \%$

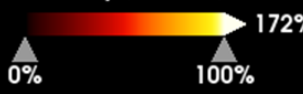




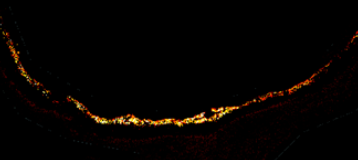

Unknown

$m / z 852.4758 \pm 6.5 \mathrm{mDa}$
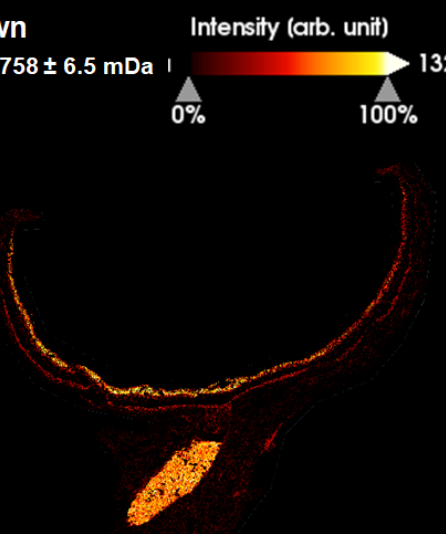

[PE(p-40:5)-H+2K] ${ }^{+}$Intensity (arb. unit)

$m / 2854.4869 \pm 6.5 \mathrm{mDa}$

$1 \mathrm{ppm}$

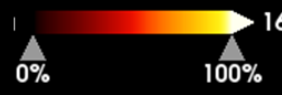
$168 \%$ $100 \%$

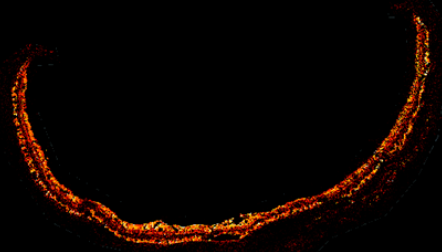

\begin{tabular}{lll} 
Unknown \\
$m / z 858.4927 \pm 6.5 \mathrm{mDa}$ & \multicolumn{1}{c}{ Intensity (arb. unit) } \\
& $160 \%$ & $100 \%$
\end{tabular}

$[\mathrm{PE}(42: 6)+\mathrm{K}]^{+}$
$\mathrm{m} / \mathrm{z} 858.5427 \pm 6.5 \mathrm{mDa}$
$2 \mathrm{ppm}$

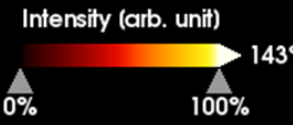

[HexCer(40:1;04)+K] ${ }^{+}$Intensity (arb. unit) $\mathrm{m} / \mathrm{z} 854.6129 \pm 6.5 \mathrm{mDa}$ $1 \mathrm{ppm}$
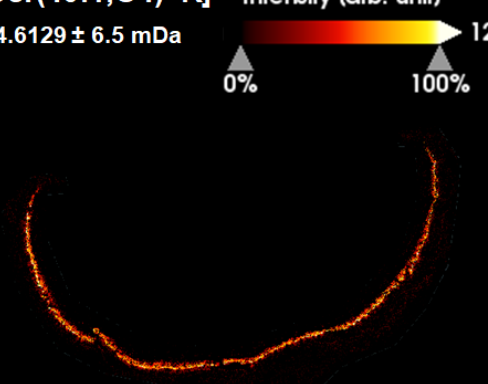

$[\mathrm{PE}(42: 9)+\mathrm{K}]+$
$\mathrm{m} / \mathrm{z}$ 852.4917 $\pm 6.5 \mathrm{mDa}$ 3 ppm

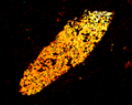

[HexCer(42:0;02)+K] Intensity (arb. unit) $135 \% \quad \mathrm{~m} / \mathrm{z} 852.6669 \pm 6.5 \mathrm{mDa}$ 2 ppm

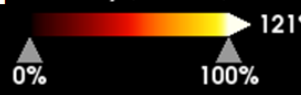

[PE(p-40:4)-H+2K] ${ }^{+} \quad$ Intensity (arb. unit) $25 \% \quad m / z 856.5028 \pm 6.5 \mathrm{mDa}$ $1 \mathrm{ppm}$ 今\% $147 \%$ $100 \%$ $1 \%$
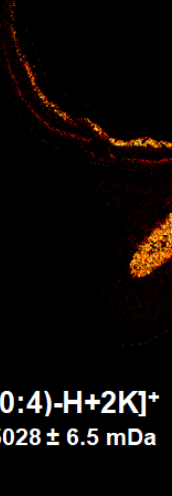

.

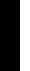



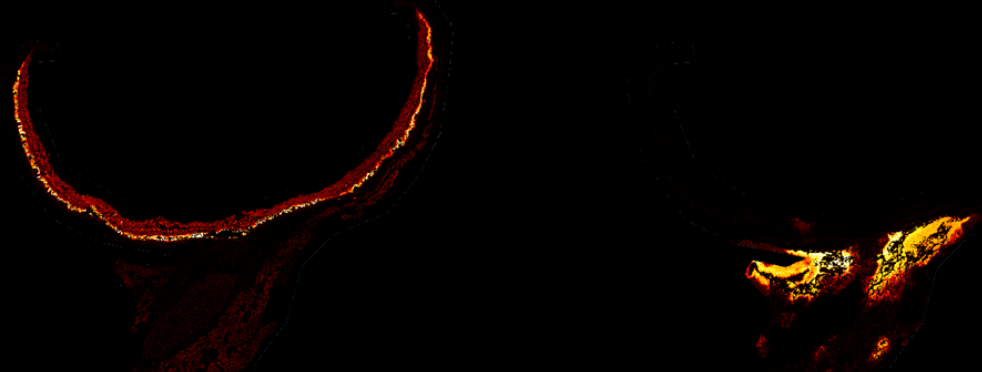

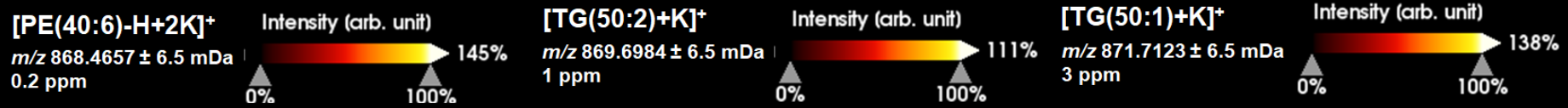

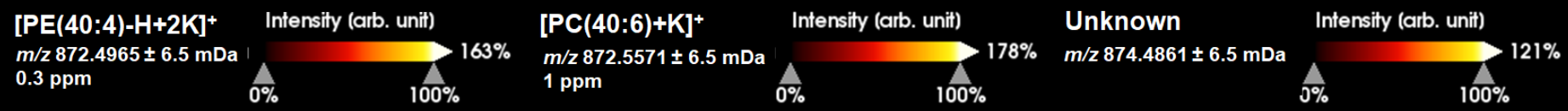

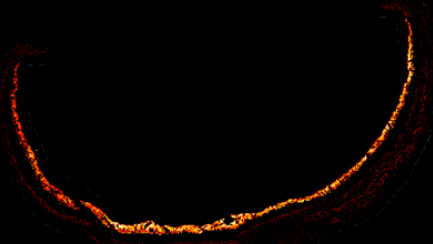

[PS(40:6)+K]+

$\mathrm{m} / \mathrm{z} 874.5007 \pm 6.5 \mathrm{mDa}$

$1 \mathrm{ppm}$

Intensity (arb. unit)

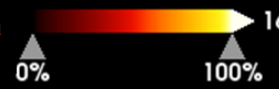

$160 \%$
[TG(52:3)+Na]+

$\mathrm{m} / \mathrm{z} 879.7414 \pm 6.5 \mathrm{mDa}$

$0.2 \mathrm{ppm}$
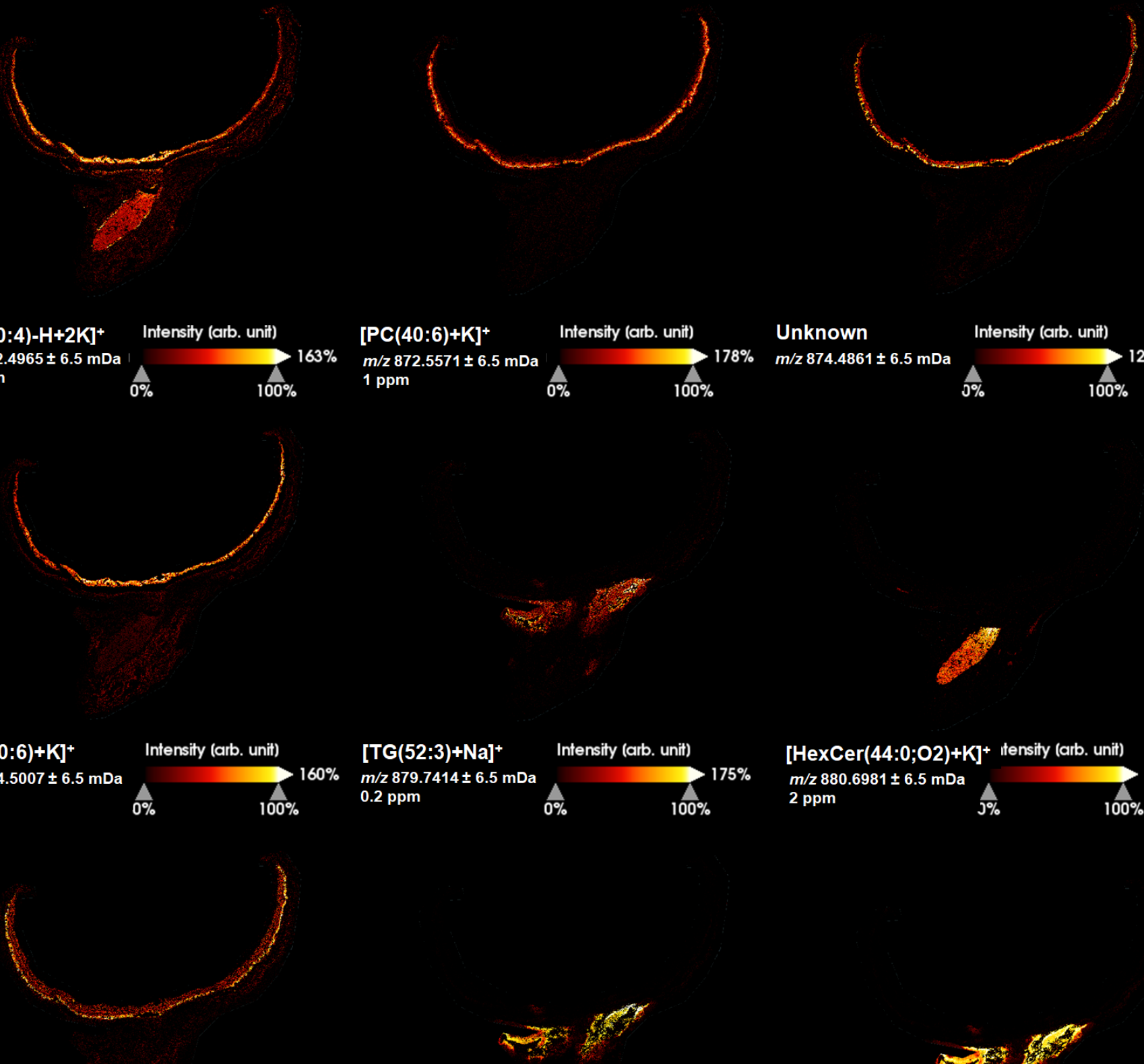

Intensity (arb. unit)

> $175 \%$
[HexCer(44:0;02)+K] Itensity (arb. unit) $\mathrm{m} / \mathrm{z} 880.6981 \pm 6.5 \mathrm{mDa}$ $2 \mathrm{ppm}$
J\%
$133 \%$
$1 \mathrm{~mm}$

Unknown

$\mathrm{m} / \mathrm{z} 882.6656 \pm 6.5 \mathrm{mDa}$

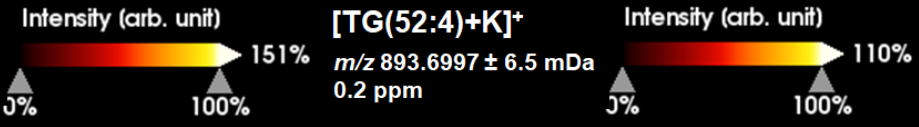

$\begin{aligned} & {[\mathrm{TG}(52: 3)+\mathrm{K}]^{+}} \\ & \begin{array}{l}\mathrm{m} / \mathrm{z} 895.7144 \pm 6.5 \mathrm{mDa} \\ 1 \mathrm{ppm}\end{array}\end{aligned}$ Intensity (arb. unit)
$112 \%$




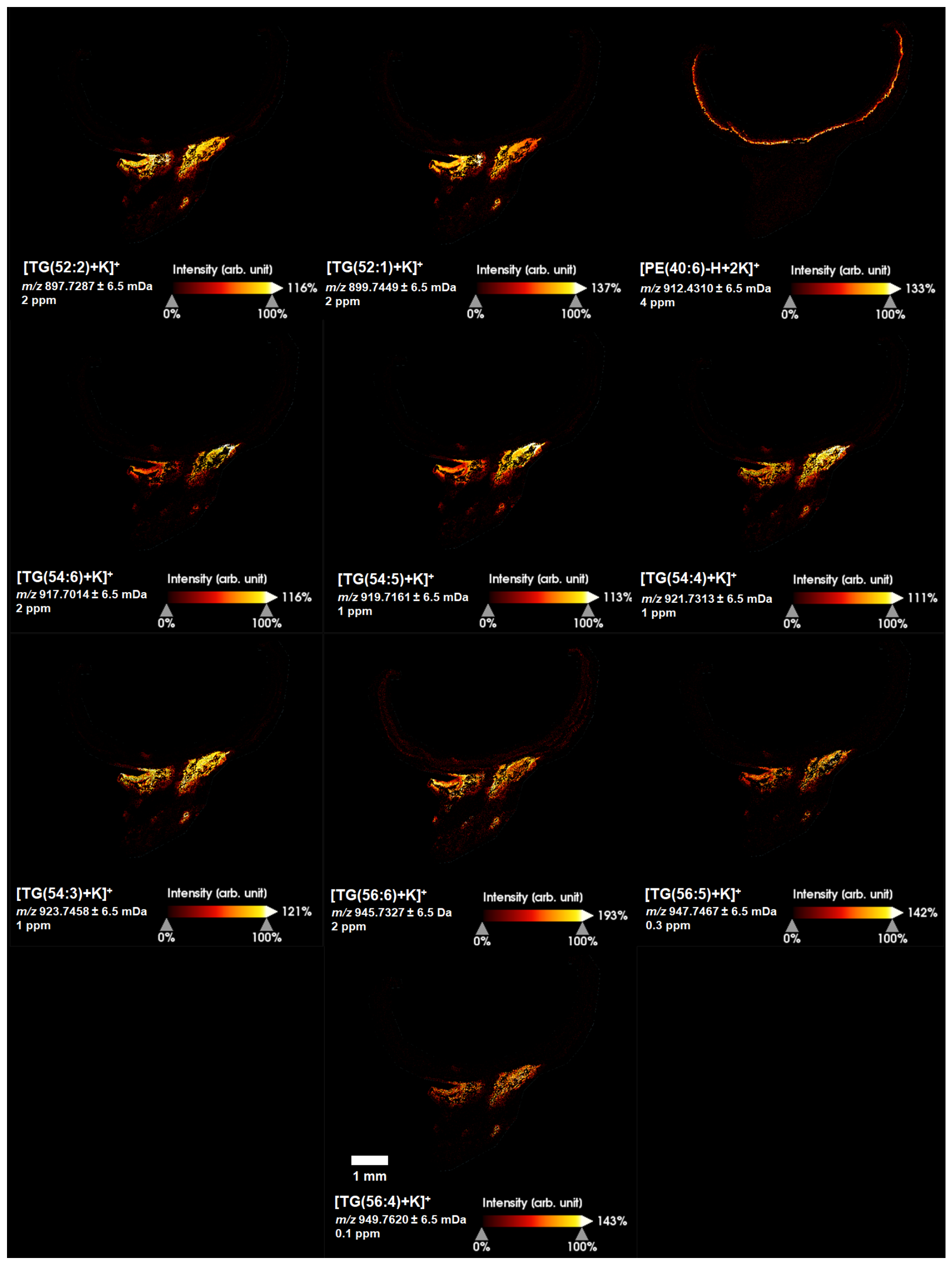


Figure S6: Collection of MALDI IMS images from an albino rat eye at 10 um lateral resolution using a precoated NAPA substrate.

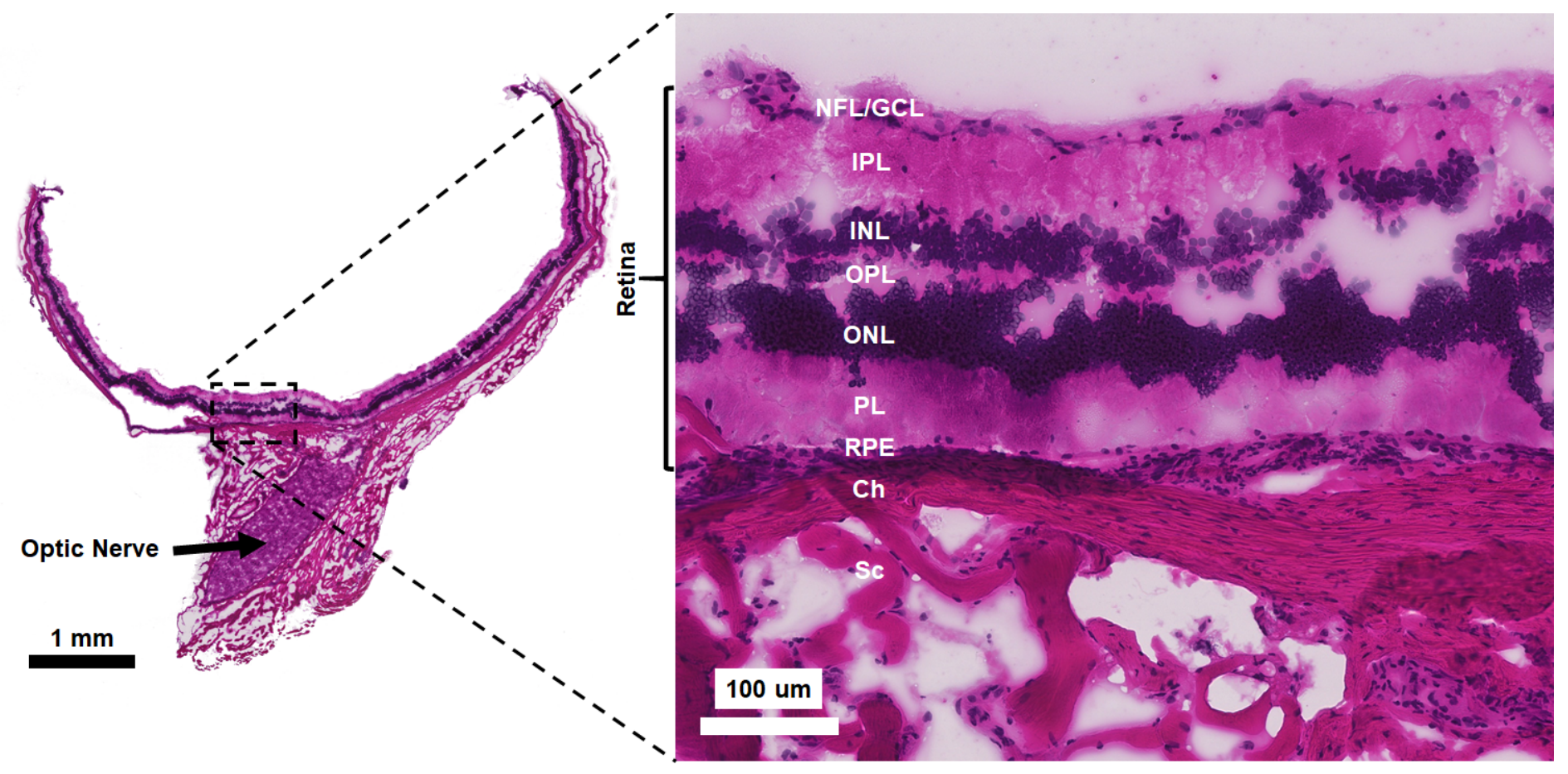

Figure S7: H\&E stained albino rat eye.

Retina:

NFL: Nerve fiber layer

GCL: Ganglion cell layer

IPL: Inner plexiform layer

INL: Inner nuclear layer

OPL: Outer plexiform layer

ONL: Outer nuclear layer

PhrL: Photoreceptor layer

RPE: Retinal pigment epithelium

Ch: Chloroid

Sc: Sclera 